\title{
La Biblioteconomía y la Documentación española en la encrucijada
}

The crisis of the Library and Information Science academia in Spain

\author{
Francisco Javier GARCíA MARCo \\ Facultad de Filosofía y Letras, Universidad de Zaragoza, C/ Pedro Cerbuna, 12, \\ 50009 Zaragoza, España, jgarcia@unizar.es
}

\begin{abstract}
Resumen
Se analiza la crisis de la enseñanza de la Biblioteconomía y la Documentación en España y se intentan desentrañar algunas de las variables que puedan estar actuando: la evolución del empleo, el impacto de los cambios tecnológicos, la reforma de la educación superior y los problemas de definición de la disciplina como ciencia. El diagnóstico es un ajuste de crecimiento de un estudios casi sin tradición en España al mercado laboral, todo ello en el marco más amplio del espectacular avance en la automatización de la información y de la globalización de la educación superior. Se recomienda mantener la atención al núcleo de los yacimientos de empleo, expandir mediante alianzas la implicación en el mundo escolar y de la gestión de contenidos, desarrollar un modelo de enseñanza de las tecnologías más eficaz, resolver el dilema entre formación generalista y especializada, reintegrar las dimensiones sociales y humanistas, enfatizar la integración teórica y discursiva y mejorar la evidencia factual necesaria para una prospectiva eficaz.
\end{abstract}

Palabras clave: Biblioteconomía y Documentación. Profesionales de la información. Gestión de la información. Ciencia de la Información. Educación. Mercado laboral. Deficiencias. Futuro.

\section{La crisis de los estudios}

La situación de la Biblioteconomía y la Documentación en España se encuentra en una encrucijada importante, marcada por el notable descenso de estudiantes nuevos en el primer curso de los programas ofertados.

En diversos trabajos a lo largo de los últimos años, Emilio Delgado López-Cózar (2002, 2003, 2007 y 2008) ha asumido la misión de dar cuenta de la evolución que han sufrido los estudios universitarios de nuestro campo durante los primeros años del siglo XXI. Los resultados, basados en los datos publicados por el Instituto Nacional de Estadística, son impactantes.

Desde el año 1998 hasta el 2006, los alumnos ingresados en la diplomatura han descendido de

\begin{abstract}
The situation of Library and Information Science teaching and academia in Spain is analyzed. The main trends analyzed are the labour market, the technological changes, the higher education reform, and the discipline definition problems. A preliminary diagnosis is an adjustment crisis to the employment market in a country with a very recent tradition in LIS education, which is proceeding in the context of the digital revolution and the process of globalization of the higher education. Some recommendations are cultivating the core employment fields, expanding with alliances the role in schools libraries and content management, developing a more realistic and effective model for technological education, resolving the dilemma between generalist and specialist education, reintegrating the human an social dimensions of the studies, emphasising the discursive and theoretical integration of the discipline, and improving the factual evidence that supports discussions about prospective trends and actions.
\end{abstract}

Keywords: Library and Information Science. Information professionals. Information management. Education. Labour market. Challenges. Future.

1524 hasta 570 , casi dos tercios. De las trece diplomaturas, tres de ellas tenían en 2006 una matrícula inferior a veinte estudiantes. Dos han desparecido ya en 2008.

La situación es solo algo mejor en la Licenciatura en Documentación. Desde el año 2000 -en el que todas las licenciaturas estaban ya en marcha salvo la de La Coruña- hasta el curso 2006-7, los alumnos han bajado de 1575 a 642. Dos programas cerraron a los pocos años de comenzar.

Con bastante retraso, la crisis alcanzó también a los estudios de tercer ciclo en 2006 . El doctorado bajó desde su techo en el curso 2005-2006 de 276 a 195 alumnos.

Igualmente, muchos diplomas de posgrado y másteres han ido cerrando, algunos comprensi- 
blemente, como los generalistas, cuyo espacio quedaba obviamente bien cubierto por la amplia oferta de estudios oficiales. En el momento actual, se está en proceso de adaptación a los másteres oficiales, de los cuales cada universidad intenta ofrecer al menos uno.

Para los que estamos en el campo académico, y que hemos realizado nuestra carrera profesional al calor de la enorme expansión de las enseñanzas universitarias de Biblioteconomía y Documentación en España que se desató a partir de su implantación en Granada en 1983, la situación llama a la reflexión.

Como todos los cambios, la situación presenta tanto ventajas como inconvenientes, y su valoración está sujeta a la perspectiva de cada cual; tanto más cuanto no todas las evidencias son negativas, como ocurre con los datos de inserción laboral respecto a otras carreras de ciencias sociales y humanas.

Sin embargo, está claro que un cambio de tendencia tan notable exige un diagnóstico, una valoración y una toma de posiciones. En realidad, lo importante no es solo clarificar lo que está pasando -aunque es necesario dibujar un panorama más preciso, basado en datos-, sino, sobre todo, preparar el futuro, y, para ello, establecer una diagnosis o, al menos, un conjunto de hipótesis que comprobar.

¿Se trata de una crisis de crecimiento, de ajuste, de tendencia o terminal? ¿Ofrece tantas oportunidades como desventajas o hay un sesgo claro? ¿Cuáles son los sectores beneficiados y perjudicados? ¿Es conveniente dejar las cosas a su curso o intentar, si es posible, actuar sobre alguna variable?

En fin, intentar prospectar el futuro de la profesión y los estudios es una tarea arriesgada, en la que es más fácil errar que acertar. Por ello, los análisis y opiniones que se presentan en estas páginas no pueden ser otra cosa que una modesta aportación a esta tarea, con todo, necesaria y decisiva.

\section{La realidad profesional en el campo de las bibliotecas}

Pero, ¿corresponde esta sensación de crisis a lo que está aconteciendo en el lado de la oferta de empleo?

Una pregunta fundamental para responder a esta pregunta es examinar cómo se está comportando el empleo de calidad para bibliotecarios y documentalistas.

A diferencia de otros sectores, es fácil examinar el empleo de calidad en las bibliotecas públicas y universitarias a través de los datos proporcionados por el Instituto Nacional de Estadística. En particular, estas estadísticas permiten observar la evolución del número de bibliotecarios con titulación específica respecto al del personal total, lo que nos permite valorar no solo la evolución del mercado laboral en estos campos, sino el impacto específico de las titulaciones universitarias en él.

En las bibliotecas públicas españolas, los resultados son positivos (tabla I). Globalmente, los puestos de bibliotecarios profesionales han ido aumentando desde el año 2000.

\begin{tabular}{|l|c|c|}
\cline { 2 - 3 } \multicolumn{1}{c|}{} & $\begin{array}{c}\text { Bibliotecarios } \\
\text { profesionales }\end{array}$ & $\begin{array}{c}\text { Total del } \\
\text { personal }\end{array}$ \\
\hline 2000 & $4.679,93$ & $17.786,26$ \\
\hline 2002 & $5.544,42$ & $17.139,54$ \\
\hline 2004 & $5.854,52$ & $18.387,57$ \\
\hline 2006 & $6.949,00$ & $20.165,47$ \\
\hline
\end{tabular}

Tabla I. Evolución del personal en las bibliotecas públicas [Fuente: Instituto Nacional de Estadística]

Existe un crecimiento importante, que se acelera justamente entre 2004 y 2006. La impresión de que el aumento del empleo está ligado al ciclo económico se refuerza al observar el bajón de 2002. Esto permite establecer la incidencia del ciclo económico, en este caso concreto de la burbuja de Internet y su impacto económico.

Hay algunos datos que son menos tranquilizadores. En particular, el número de puntos de servicio, que también había descendido algo entre 2000 y 2002, disminuye de 8099 en 2004 a 8.066 en 2006 . Sucede lo mismo con el número de bibliotecas, que lo hace de 6.595 a 6.523 .

En las bibliotecas universitarias también se observa un crecimiento importante: muy fuerte durante los años noventa y notablemente menor para el tramo 2000-2006 (tabla II). El personal en general crece más que los profesionales, ambos especialmente hasta el 2002. Los profesionales lo hacen a una tasa mayor entre el 2000 y el 2007 . Entre el 2002 y el 2006 las cifras se estabilizan, aunque fluctúan ligeramente en lo que respecta al personal en total. Las tasa de crecimiento mejora algo del 2006 al 2007.

\begin{tabular}{ccc}
\hline Años & $\begin{array}{c}\text { Bibliotecarios } \\
\text { profesionales }\end{array}$ & Personal (EJC) \\
\hline 1994 & $1.650,00$ & $4.352,00$ \\
1995 & $1.629,00$ & $4.523,00$ \\
1996 & $1.711,00$ & $4.846,00$ \\
1997 & $1.849,00$ & $5.564,00$ \\
1998 & $1.879,00$ & $5.495,00$
\end{tabular}




$\begin{array}{lll}1999 & 2.014,00 & 5.890,00 \\ 2000 & 2.141,00 & 6.052,00 \\ 2001 & 2.168,00 & 6.294,00 \\ 2002 & 2.266,00 & 6.549,00 \\ 2003 & 2.300,00 & 6.642,00 \\ 2004 & 2.328,00 & 6.756,00 \\ 2005 & 2.361,00 & 6.681,46 \\ 2006 & 2.387,28 & 6.654,32 \\ 2007 & 2.465,75 & 6.791,00\end{array}$

Tabla II. Evolución del personal en las bibliotecas universitarias [Fuente: Instituto Nacional de Estadística]

Las cifras son buenas respecto al empleo público en general, que se incrementó entre 2000 y 2007 en su conjunto un 9,01\% (Ministerio de Administraciones Públicas, 2008), frente al $15,16 \%$ de los bibliotecarios universitarios. Los datos de los que disponemos para bibliotecas públicas, que llegan hasta 2006, son incluso un poco mejores.

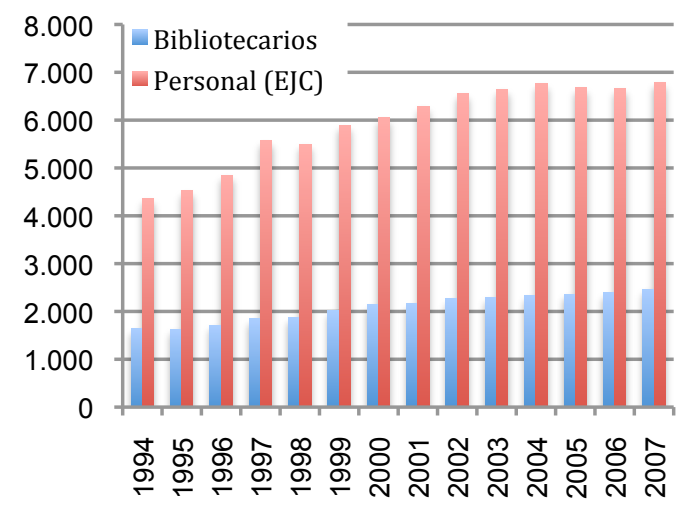

Gráfico 1. Bibliotecarios y personal a tiempo completo en las bibliotecas universitarias [Fuente: REBIUN]

Así pues, la impresión que transmiten estas cifras no es de crisis, sino de un importante crecimiento durante bastantes años, que ha conducido a un mercado laboral maduro y estabilizado en 2006 y 2007. Es importante añadir, con todo, que se ha fracasado hasta el momento en incorporar los bibliotecarios de carrera al mundo de las bibliotecas escolares.

\section{Un acercamiento al ajuste de la oferta de titulados a la demanda laboral}

Si sumamos los egresados de la diplomatura y la licenciatura en el año 2006, obtenemos una cifra de 1065 personas.
Por el contrario, si sumamos los puestos de bibliotecarios profesionales titulados en las bibliotecas públicas y universitarias, se alcanzan 9.336 puestos de trabajo, los cuales, sobre una vida laboral media de 35 años, permite calcular la creación vegetativa de unos 266 puestos de trabajo anuales.

Si tenemos en cuenta que el empleo en bibliotecas se puede estimar en alrededor del $35-40 \%$ del sector basándonos en los estudios más recientes (Borrego, Comalat y Estivil, 2004; Moreiro y colaboradores, 2008), se puede calcular que la demanda media anual de empleo de calidad fijo podría estar en una horquilla de entre 667 y 762 personas anuales.

Este número puede mejorar algo si se contempla la tasa del diferencial de crecimiento entre el mercado laboral estatal y privado. Los datos manejados por Moreiro, Tejada y colaboradores dan un $79 \%$ del empleo ligado a las administraciones para la promoción de 1991 (Moreiro, Moscoso y Repiso, 1995), un 47,7 \% para la de 2000 (Tejada y Moreiro, 2003) y un 43, $4 \%$ en 2006 (Moreiro et al., 2008). Por otra parte, estos datos tienen una lectura positiva en cuanto al crecimiento de empleo fuera de la administración pública, pero también podrían indicar una tendencia a un límite en torno al 60 por cien para el empleo fuera del Estado.

Además, como bien se ha señalado, los datos de inserción laboral son bastante buenos comparados con otras carreras de Humanidades y Ciencias Sociales, y la disminución del número de alumnos puede ayudar a hacer esta relación óptima, sentando las bases de una recuperación de la matrícula.

Así pues, los datos de empleo en el campo de las bibliotecas transmiten un moderado optimismo.

\section{El diagnóstico: una crisis de ajuste}

El diagnóstico provisional a la vista del contraste entre los datos de matrícula y los datos de empleo es que se trata de la primera crisis de ajuste de crecimiento que se ha producido en España para los estudios. Al ponerse en marcha unos estudios nuevos sin información real de cómo respondería el empleo, se ha tenido que producir un ajuste entre ambas variables.

El hecho de que la disminución de matrícula haya sido mucho menor en la Diplomatura de Barcelona, que son los estudios con mayor tradición en España, avala esta hipótesis. De 162 alumnos de nuevo ingreso en 1998, se pasó a 139 en 2006. La licenciatura de la Universidad 
de Barcelona, sin embargo, se ha comportado de forma mucho más próxima a la media.

Además, dado que Biblioteconomía y Documentación, a diferencia de otras carreras, se estudia claramente con una perspectiva profesional muy definida, tiene especial sentido que la demanda se vaya ajustando a la oferta según ésta se va clarificando. A diferencia de carreras como Filosofía o Arte, Biblioteconomía y Documentación raramente se estudia con perspectiva únicamente cultural y de desarrollo personal.

Estas conclusiones son consistentes con otros estudios realizados en el entorno internacional. En particular, Andrew Dillon y April Norris (2005) resumían así su análisis de los programas de información y documentación en Estados Unidos:

In the present essay we examine the major claims for a new crisis in LIS education and conclude that the data do not support most of the popular criticisms made of this field. Instead, the notion of crisis is best understood as indicative of a moment of change and an opportunity to significantly affect the long-term future of the field.

[...] The crisis we face is less to make research more relevant to local concerns of practitioners, or to revamp once more a set of core classes or accreditation standards, but to demonstrate our authority as a profession in dealing with information issues at both theoretical and practical levels, within academia and beyond. This is a crisis in the sense of it being an opportunity and a turning point, and in that sense, it should be welcomed, if we truly believe we, as a profession and a discipline, have something substantial to offer.

\section{Más allá de la crisis de matrícula}

No obstante, es necesario seguir prestando atención al estacancamiento en el crecimiento de los puestos de trabajo que se produce en el 2006-2007, por si refleja un cambio de tendencia y problemas de fondo más graves.

De hecho, el escenario tranquilizador que hemos obtenido a partir de los datos de empleo en bibliotecas es cuestionado por las discusiones públicas que se han venido realizando en diversos foros profesionales.

El debate realizado en la lista IWETEL a raíz del mensaje enviado por Emilio Delgado (2007) el siete de octubre de 2007 -y cuya cola se extendió hasta el 24- sobre el Ocaso de las enseñanzas universitarias de Documentación en España es una buena muestra de las principales posiciones. La impresión general es de cuestionamiento del status quo, aunque aquéllas son muy variadas. Datos fragmentados, perspectivas y modelos diferentes, e, incluso, talantes ante la vida explican las diferencias (Anuario, 2007).

Las repuestas muestran los desgarros de la profesión y de la academia: entre la tecnología, las ciencias sociales y las humanidades; entre profesionales y académicos; entre jóvenes con empleo precario y profesionales bien establecidos; entre investigadores y docentes; entre los diferentes nichos profesionales; entre gente con formación específica y personas que acceden con títulos más generales...

Sea justo o injusto, está claro que muchos estudiantes, profesionales y académicos ven las bibliotecas como un entorno que no crece adecuadamente y sometido a incertidumbres importantes. Esta realidad se ha visto reflejada en el cambio de nombre de los estudios, que abandonan el término "biblioteca" o "Biblioteconomía" a favor de "Información y Documentación".

En las páginas siguientes vamos a intentar aproximarnos a ese conjunto de retos que ponen en cuestión la trayectoria de estos últimos años que, con sus luces y sus sombras, puede calificarse claramente de positiva.

\section{Los retos: otras crisis en el horizonte}

Como hemos visto, la hipótesis del ajuste y los datos que la avalan sirven para explicar la evolución de la matrícula y la situación actual. Sin embargo, hay una cierta intranquilidad en el ambiente que se refleja en algunos debates públicos.

Por otra parte, si las condiciones en curso cambiaran de forma importante, el escenario actual no tendría validez. Es necesario, por tanto, prospectar los factores de cambio y ver que implicaciones pueden tener para el futuro

\subsection{La encrucijada tecnológica}

El factor más importante de cambio estructural que se encuentra en marcha es, sin duda, la revolución de las tecnologías de información y comunicación, en particular, de la Internet y la World Wide Web.

Como hemos visto, las bibliotecas y otros centros de información y documentación se han beneficiado extraordinariamente de la primera fase de la informatización, y, de hecho, han crecido en personal y recursos.

Sin embargo, la nueva ola de cambio provocada por el surgimiento de gigantescos servicios en la Red que utilizan las TIC para generar una experiencia de comunidad virtual -la web 2.0- y por la creciente automatización de la información y la comunicación en la web -la Web 
Semántica- plantean retos de cariz completamente distinto.

El primer reto afecta a la actual configuración de los servicios de información y documentación, centrados en la gestión del papel y el celuloide en almacenes, catálogos y servicios de consulto: Gracias a la World Wide Web, el acceso al documento completo a través del ordenador asegurando la interacción social es una alternativa real al uso de espacios físicos.

El segundo reto amenaza a la manera en cómo se realiza el trabajo informativo-documental en la actualidad: La automatización del procesamiento de la información y la documentación digital que plantea la Web Semántica plantean a medio plazo una amenaza radical a la estructura actual de los servicios de información y documentación, basada en un trabajo de carácter fundamentalmente artesanal.

Efectivamente, gran parte de las técnicas inventadas en el seno de nuestra disciplina - como la representación de documentos para la recuperación y el control de vocabulario- no tienen una gran complejidad y pueden ser asimiladas con facilidad relativa por cualquier cuerpo profesional. El énfasis se sitúa en la actualidad en la automatización de sus procesos, y para ello hace falta una formación y una disciplina propias de las ciencias y tecnologías "duras". Incluso las tareas que todavía no pueden hacer las máquinas, como catalogar y clasificar, van siendo abordadas o circunvenidas. Pensemos tan solo que la posibilidad de la catalogación derivada o en ejemplar, eliminan procesos que antes había que hacer en cada centro. Igualmente, la combinación de búsqueda probabilística y basada en citas que realiza Google permite resolver bastante bien las consultas específicas y de una manera aceptable muchas necesidades de información temáticas.

Estos retos no pertenecen al mundo de la imaginación y los temores inciertos. Las grandes empresas del mundo digital -Google, Microsoft, Yahoo, Amazon, Apple...- están teniendo éxito en el proceso de crear grandes repositorios de información totalmente automatizados. Repositorios como iTunes, Google Scholar, Google Maps, Google Books, Amazon Search Inside, Flickr, TyouTube, etc., son botones de muestra del futuro que viene y que, en cierta manera, ya está aquí. Estas grandes empresas se preocupan actualmente sobre todo del mercado de consumo general, pero ya han iniciado incursiones en el campo de la información académica y seguirán sin duda en otros campos.
En este contexto, el sistema educativo español está respondiendo de una manera muy conservadora, especialmente si se le compara con iniciativas norteamericanas como el movimiento de las iSchools. Probablemente, la universidad se encuentra mal equipada para este nuevo reto: el cuerpo de profesores ha envejecido notablemente, y su crecimiento, como señala con cifras Emilio Delgado (2007) se encuentra estancado.

\subsection{Crisis de expectativas}

Lo cierto es que la revolución informática primero y de las telecomunicaciones después ha potenciado enormemente en recursos, puestos de trabajo y prestigio social a las profesiones de archivero, bibliotecario y documentalista, pero no ha resultado, como algunos pronosticaban, en la asunción de un papel estelar dentro de la sociedad del conocimiento.

Esta situación golpea con fuerza un lugar común de la autoimagen de los profesionales de la información y la documentación: aquél que nos sitúa como capitanes de la Sociedad de la Información.

Esta era, sin duda, una imagen excesiva. La Sociedad de la Información no pertenece a los profesionales de la información y la documentación; sino que, por el contrario, forman parte de ella. Podemos aspirar a tener un papel importante en ella, pero la gestión de la información es cuestión natural del conjunto de las profesiones; y todos los que aspiran a una posición de control y dirección dentro de las organizaciones desarrollan un discurso relacionado con la información y su gestión. Un caso ejemplificador lo constituye la gestión del conocimiento - una disciplina que pretende ocuparse específicamente de este aspecto. En ella, encontramos profesionales de casi todas las ramas de la gestión, desde los recursos humanos, a la ingeniería informática, pasando también por el derecho y la documentación, por supuesto.

Por otra parte, la revolución de Internet ha proporcionado muchas oportunidades, pero también ha minado la confianza en el futuro. Bajo el impacto de la Internet y, especialmente, de Google, muchas personas (profesionales y docentes) han dejado de creer en las unidades, servicios y procesos tradicionales de la Biblioteconomía, la Archivística y la Documentación.

Es cierto que, ante la inminencia de una biblioteca digital universal y quizá de grandes gigantes de la distribución documental, no se puede estar como si no pasara nada. Pero la solución 
no es centrarse en las competencias meramente tecnológicas. Es necesario tecnologizarse, pero hacerlo "destecnologizándose", marcando una diferencia con las profesiones tecnológicas, mudando los enfoques a los aspectos sociales, los servicios de proximidad, la gestión del patrimonio... Creo que el énfasis no estará en el futuro en la tecnología, sino en la interacción social y personal en entornos tecnológicos. Como señalan varios autores, la Web Social constituye un horizonte prometedor, pues une los dos contextos, aunque difícilmente agota el mundo de la información y la documentación.

\subsection{La crisis disciplinar}

Desde la segunda guerra mundial, el mundo de la información y la documentación ha conseguido configurarse como un grupo visible y respetado de comunidades de investigación, pero no ha cristalizado en una ciencia "normal" en la terminología de Kuhn. El resultado de ello es una permanente crisis disciplinar, en la que actualmente destacan dos frentes.

El primero, el de la definición de la disciplina al que podríamos denominar "ontológico"-, tiene que ver con la dificultad de conseguir una disciplina integrada, más allá del mosaico de campos, paradigmas y prácticas profesionales y científicos que conforman las "Ciencias de la Documentación".

El segundo es de carácter metodológico - si se quiere, "epistemológico" - y tiene que ver con la pluralidad de métodos de investigación utilizados. No existe un paradigma único que aborde todo el espectro de problemas de investigación.

Como resultado, coexisten varias disciplinas deslavazadas y, muchas veces, el campo se estudia desde disciplinas externas distintas.

\subsubsection{El problema ontológico}

En los últimos años, la Biblioteconomía y la Archivística han crecido para incorporar respectivamente a la Documentación y la Gestión de Documentos; sin embargo, permanecen en gran parte de espaldas la una a la otra. En nuestro país, solo encuentran un modus vivendi cómodo cuando comparten de forma confederal asociaciones profesionales y titulaciones. Esto es adecuado en el mundo profesional, pero en el científico es una situación casi mortal porque, a pesar de las soflamas, el mundo de la información documental no se visualiza como un campo disciplinar sólido e integrado.
La esperanza de que la Information Science pudiera proporcionar esa base común se está esfumando rápidamente, conforme, muerta de éxito, se "comoditiza" a toda velocidad dentro de la ingeniería informática, al convertirse en la base de la industria de los buscadores de Internet. Esto es cierto, especialmente, de su campo más fructífero, la recuperación de la información (no como práctica, sino como teoría y paradigma de investigación). Como resultado de las últimas tendencias, la parte de ciencia "dura" de la Biblioteconomía y la Documentación está en buena parte abandonando la disciplina.

Así pues, la biblioteconomía y la documentación siguen treinta años después constituyendo un "terreno intelectual dividido" (Dillon, 2007). Por suerte, sin embargo, también las preguntas fundamentales que definen el campo conservan toda su actualidad: qué es la información "a largo plazo" (1), cómo se usa y mal usa, cómo se puede utilizar para bien de las personas, las organizaciones y las sociedades, cuáles son los retos en este momento qué afrontar dentro de esta tarea.

\subsubsection{El problema metodológico}

Un problema importante en nuestra disciplina es que no es tan solo un campo de investigación, sino de decisión y acción. Parte de la decisión y la acción se realizan con métodos y tecnologías probadas científicamente - esto es, con datos-, pero en los campos en los que no existe "evidencia dura" las decisiones se toman a partir de un esquema degradado de opciones cada vez más alejadas del método científico: discursos sistemáticos, razonables; enfoques basados en la afiliación ideológica; y, en último término y si no existe otra opción, en la mera opinión.

Los paradigmas de investigación "duros" que existen en las Ciencias de la Documentación son la recuperación de la información experimental y los estudios bibliométricos (incluyendo la florenciente webmetría, en la que tenemos investigadores notables). Otro paradigma más inestable en cuanto a metodlogías y contenidos son los estudios de usuario, que en los últimos años están siendo absorbidos dentro de la recuperación de la información (Åström, 2007). Fuera de estos paradigmas persisten un conjunto de metodologías estructuralistas $\mathrm{y}$, por supuesto, un amplio elenco de perspectivas filosóficas e ideológicas que producen discursos académicos coherentes, pero que están quedando en la perifería de lo que se considera investigación, toda vez que el campo de investi- 
gación ha sido inscrito dentro de las ciencias sociales.

Hay un campo enorme para los estudios antropológicos, sociológicos, politológicos y económicos en el campo de la información, que no son tocados sino de forma sintética y con objetivos de discurso ideológico y toma de decisiones, bien entendido que son usos legítimos y necesarios.

En los últimos años, la investigación sobre recuperación de información y automatización de bibliotecas y archivos está ligada a Internet; se hace sobre todo en el sector privado - por ejemplo, en Google, Yahoo o Microsoft-; y es cada vez más una actividad desarrollada por ingenieros informáticos. De hecho, los ingenieros informáticos han visto futuro en el campo y se han subido al carro de la práctica profesional, académica e investigadora en la Information Science (IS). Por otra parte, es necesario recordar que muchos de los "padres" de la IS eran ingenieros o científicos no sociales.

En fin, el campo está dividido en cuatro frentes que se trabajan con métodos procedentes de disciplinas muy distintas: el desarrollo de sistemas - orientación tecnológica-; la gestión de sistemas -orientación administrativa-; el enfoque en el usuario -orientación social-; y el estudio de las fuentes o documentos - orientación histórica y humanista-. Los cuatro campos son importantes, pero se cultivan con métodos muy diferentes.

Al igual que ocurre con el campo profesional, la automatización conlleva tanto las mayores amenazas como las mayores oportunidades para el desarrollo de la investigación en el área.

Dado el lugar central de la automatización, a largo plazo, la investigación social y humanística que tendrá relevancia será la que sea capaz de incidir en el desarrollo de los modelos y herramientas tecnológicas $\mathrm{o}$, secundariamente, en las organizaciones que las utilizan.

Sin embargo, en el lado del haber, el factor tecnológico ha propiciado un fuerte movimiento normalizador dentro del campo, fruto del cual el vocabulario y los enfoques entre las distintas subdisciplinas se han acercado considerablemente. Como consecuencia, cada vez hay más elementos de estudio comunes a las distintas disciplinas: tecnologías, gestión, formatos de documentos, etc.

\subsection{La encrucijada educativa}

Otra crisis de gran calado que se encuentra ahora en marcha es la que afecta a la educación superior en su conjunto, y en la cual la Biblioteconomía y la Documentación tiene que encontrar un espacio específico para sobrevivir.

En nuestra opinión, la encrucijada educativa está marcada por un proceso a largo plazo - la maduración de la industria de la educación superior en occidente-, otro de carácter regional -el proceso de convergencia europea-, y otro específico de los estudios de bibloteconomía y documentación, que tienen que interactuar en ese entorno con una visibilidad y unos recursos muy limitados.

6.4.1. El aumento de la competencia entre instituciones y programas de enseñanza superior

Tras décadas dedicadas a desarrollar la educación superior, está ganando fuerza entre la clase política europea la opinión de que se trata de una industria madura, y que se puede y se debe dejar parcialmente a la libre competencia. El objetivo es acercarla al modelo mixto norteamericano: universidades de élite -generalmente privadas- que compiten a nivel mundial y una red de universidades suficientes, generalmente públicas, algunas también muy bien situadas en el ranking mundial.

En primer lugar, esto supone permitir la apertura de universidades a otros agentes distintos del Estado. En una segunda fase, este proceso implica favorecer la competencia entre las universidades y los programas que éstas ofrecen, sean públicas o privadas; y en ese momento nos encontramos actualmente en España. En general, las universidades estatales españolas han terminado por monopolizar las enseñanzas de información y documentación, y los agentes privados han abandonado la gran mayoría de los programas iniciados. Esto tiene que ver, como hemos visto, con el sobredimensionamiento de los estudios respecto a la creación de empleo de calidad, y, en parte, es una mala noticia porque los agentes privados suelen estar por lo general más orientados al mercado.

Otro pilar importante es la diversificación y especialización de la oferta educativa. Se está buscando desde años una formación cercana a las necesidades del mundo del trabajo, lo cual ha llevado a una enorme apertura de la oferta de enseñanza para dar cuenta de los diferentes nichos de empleo. En nuestro país aun queda camino por recorrer, pero se está cubriendo rápidamente gracias a los nuevos decretos de posgrado y grado. 
En cualquier caso, la dinámica actual de los estudios universitarios en una dinámica liberalizada es absolutamente distinta a la anterior, fuertemente regulada. Ahora no es posible hacer con los planes de estudio y los estudiantes ingeniería social o extraer únicamente un vector que resulta de los intereses y de la dinámica social del cuerpo de profesores, porque no hay un monopolio de la enseñanza. Es necesario atender a los nichos profesionales concretos, al público objetivo y a los estudiantes reales que atienden a los estudios. Si se pierde el contacto con los yacimientos de empleo y de dotaciones de recursos, la suerte está echada.

\subsubsection{La apuesta por el cambio de nombre y el problema de las competencias nucleares}

En este mar de nuevas titulaciones y con un escenario de casi pleno empleo nuestras profesiones se desdibujan, y pierden visibilidad, atrayendo tan solo a personas que están realmente interesadas.

Como no puede ser de otra manera, la mayor parte de los expertos ponen su fe en la multiplicación de las enseñanzas relacionadas con las nuevas tecnologías, y la nuestra tampoco es diferente en eso. Sin embargo, en un análisis somero se puede observar que la proporción de créditos especialmente dedicados a la formación en tecnologías de la información sobre el total de los estudios no se ha incrementado desde los primeros planes de estudio; $y$, en algunas universidades, ha disminuido ligeramente. Cuestión distinta es que ahora las tecnologías de la información y la comunicación permean el conjunto de las asignaturas, pero sin capacitar en aspectos más específicos como la programación o el mantenimiento de sistemas.

Otro aspecto relacionado con la competitividad de los estudios que ha sido muy debatido es la marca, esto es, el nombre de los estudios. El sector innovador ha rechazado el nombre Biblioteconomía, que es cierto que en la calle no se entiende muy bien, $y$ los profesionales $y$ académicos de otras disciplinas de las ciencias de la documentación han apoyado también un nombre más genérico.

La solución que se ha adoptado es denominar a los nuevos grados en "Información y Documentación". En España se ha vivido como un triunfo respecto a las anteriores reivindicaciones frente a las disciplinas de la comunicación, que habían "monopolizado" el concepto.
Es cierto que el nombre anterior era desafortunado porque no era bien comprendido, pero el nuevo supone una extensión excesiva de la intensión de la disciplina. También es cierto que se sigue la estela de movimientos importantes en el entorno internacional.

En el lado de los contra, está claro que identificar a la biblioteconomía, la archivística y la documentación con el mundo amplísimo de la información es un exceso a todas luces, semejante a aquél en el que incurrieron las facultades españolas de comunicación, también necesitadas de un nombre colectivo que incluyera a periodistas y publicistas. Además, más pragmáticamente, la opción abre el paso a qué, cuando apriete la crisis de titulados en Información y Documentación y se supere la todavía en curso expansión de los titulados en comunicación, se decida la integración de muchos programas, como ha sucedido en el Reino Unido.

En el lado de las ventajas, está claro que supone incrementar el campo de acción. En Estados Unidos, existe un movimiento muy fuerte hacia el concepto de información, conocido como iSchools. La valoración de Blaise Cronin (2005) es muy positiva, aunque reconoce lo excesivo del nombre:

What can be said with assurance, however, is that as a result of recent structural rumblings and readjustments, the discourse space we occupy has been enlarged (a healthy enough development in itself) even if the accompanying rhetorical excesses and ontological legerdemain leave something to be desired. For now, our identity crisis continues to flourish.

Se trata, en fin, de un factor de difícil valoración. Solo el tiempo dirá si el énfasis en la información ha sido un éxito, un ejercicio de voluntarismo o un error manifiesto.

En cualquier caso, para sustentar una profesión, no se trata solo de hacer cosas interesantes, hay que mantener fronteras claras con las demás, con un núcleo de competencias que se hacen mucho mejor que otros. No basta decir que hacemos cosas interesantes en el campo de la información, sino también demostrar que cosas hacemos mejor que otros.

Mi opinión es que la preservación y representación del conocimiento registrado y la mediación en recuperación de ese conocimiento (incluyendo aquí la referencia) son, junto con la mediación de información, los aspectos centrales. Durante bastante tiempo requerirán en muchos sitios un trabajo intelectual y de interacción personal, aunque, por supuesto, con fuerte apoyo de las tecnologías. Eso crea un espacio sufi- 
cientemente diferenciado del desarrollo y mantenimiento de sistemas, un área en la que estamos en desventaja colectivamente frente a otras profesiones.

Progresar con los cambios tecnológicos es absolutamente necesario, pero no es una respuesta por sí sola. Las tecnologías son precisamente lo que a otros profesionales se les da igualmente bien o, como mucho, a veces mejor y a veces peor: ingenieros informáticos, programadores, maestros, especialistas temáticos. La aporía, además, está servida: tecnología de la información y la comunicación, pero sin saber programar... (Eito, 2007; Perez Aguera, 2008).

\subsubsection{El efecto Bolonia}

La tendencia más importante en el entorno español, que se ha recibido en general como esperanzadora, es, sin duda, el proceso de convergencia europea y la aprobación de los primeros grados y posgrados conforme a las nuevas directrices.

El efecto Bolonia ha servido de catalizador de muchos cambios positivos - fundamentalmente, un avance del aprendizaje activo y supervisado-, pero también ha producido una reorientación del trabajo de los académicos que puede ser perjudicial en un campo como el nuestro en el que lo que más falta es investigación, no más compromiso docente y de transferencia, campos en los que las titulaciones que funcionan ya destacan.

Ciertamente en una ecología del saber compleja como la actual, la investigación y el desarrollo son siempre interdisciplinares, y existe una división del trabajo entre la creación y difusión del conocimiento; pero una disciplina sin investigación está condenada a la desaparición.

\subsubsection{Una disciplina con escasos recursos}

Otra debilidad importante tiene que ver con las profesiones y los académicos en España con respecto a otras disciplinas.

Respecto a nuestro papel en la ecología de las Ciencias Sociales, de ninguna manera nos podemos comparar en tamaño, influencia y recursos gestionados con las Ciencias Económicas, Derecho o Comunicación, por mencionar algunos de los grandes.

\subsection{El cambio de ciclo económico}

El reto a corto plazo más importante es el cambio dentro del ciclo económico mundial y español.
Desde mediados de 2007 ser veían signos de agotamiento del actual ciclo de crecimiento, que ha sido particularmente largo. En estos momentos, lo único que está en cuestión es su duración y profundidad, con analistas que plantean escenarios suaves, con una contracción mínima y una duración de dos semestres, hasta otros que hablan de una estanflación duradera.

Una consencuencia inmediata está siendo el aumento del paro, que, paradójicamente, puede ser coyunturalmente beneficioso para los programas de estudio al favorecer la afluencia a las aulas de parados y jóvenes en busca de un primer empleo.

Otra consecuencia es que el Estado se va a quedar rápidamente sin dinero para acometer proyectos en el campo de la cultura y la educación. Esto es debido a tres factores: la contracción del crédito por la crisis del sistema bancario internacional; la disminución de los ingresos del Estado y las Comunidades Autónomas debido al descenso de las transacciones inmobiliarias y el consumo; y el aumento de los gastos sociales del Estado en prestaciones y subsidios de desempleo y sostenimiento de las pensiones en una situación de inflacción. Sin dinero para cultura y educación, las bibliotecas y archivos pueden sufrir incluso una contracción, que ya se apreció en los años siguientes a la explosión de la burbuja tecnológica en el año 2000.

Desgraciadamente, este escenario nos retrotrae a los años de despegue de la titulación, caracterizados por el crecimiento de los estudiantes, los escasos medios docentes y el paro y subempleo de los titulados.

\section{Algunas líneas de acción}

A la vista del análisis realizado, es posible recomendar algunas líneas de acción.

7.1. Mantener el centro y desarrollar con humildad y en colaboración la periferia

El núcleo principal del empleo -entorno al $40 \%$ como mínimo- sigue estando en las bibliotecas y centros de documentación. Los estudios de nicho, especialmente en el campo de los archivos y la gestión de documentos disfrutan de un empleo reducido, pero estable; a la espera del fruto que sin duda dará el esfuerzo que se está produciendo a nivel internacional en el campo normativo. Es necesario atender con rigor $y$ calidad a estos núcleos de empleo.

Es necesario también afrontar de forma realista el tema de las bibliotecas escolares, probablemente a través de estudios conjuntos con magisterio, como un área interdisciplinar. A día de 
hoy, es uno de los grandes fracasos de la disciplina, y quizá sería mejor aspirar a un poco y conseguirlo, que posiciones maximalistas que solo se pueden abrir camino, hoy por hoy en España, con una partida presupuestaria del Estado que la crisis aleja y que el énfasis en la Internet hace casi imposible.

En la periferia, la gestión de contenidos ofrece grandes posibilidades de futuro. El problema de esta propuesta es que la gestión de contenidos, al menos en España, está quedando más bien del lado de la Ingeniería Informática. No podemos olvidar que el grueso de los alumnos de Biblioteconomía y Documentación se reclutan en los bachilleratos de Humanidades y Ciencias Sociales, y que estos estudiantes están mayoritariamente interesados en su gran parte... por el mundo del libro, los documentos, las bibliotecas y los archivos, y raramente por los sistemas de información, que parecen a una persona de dieciocho años cuestión de la Ingeniería Informática.

La gestión de contenidos aparece como un campo atractivo, pero en el que hay que competir y colaborar con los profesionales de la informática, y esperar que cada grupo vaya encontrando su lugar, que, por la naturaleza del campo, difícilmente será estable.

\subsection{Necesidad de desarrollar un modelo} de enseñanza de las tecnologías eficaz

Hay que terminar con el "desencuentro" (Moscoso, 2000) entre las disciplinas clásicas de la Biblioteconomía y la Documentación y la Informática, y potenciar la formación técnica (Martínez Méndez, 1997).

El problema de enseñar muchas nuevas tecnologías a personas que no tienen un interés importante en ellas o una preparación suficiente es complicado: dar mucho requiere mucho tiempo; dar poco es quedarse a nivel de introducción. La primera opción excluye contenidos de otras asignaturas, muchas veces más centrales al nicho de empleo que se pretende ocupar. La segunda opción supone proporcionar un nivel de formación que difícilmente se puede considerar profesionalizante.

No obstante esta aporía de difícil solución, se pueden dar pasos correctos.

El primero es integrar definitivamente la tecnología informática en la enseñanza de todas y cada una de las disciplinas, aunque lógicamente en distinta medida.

En segundo lugar, es conveniente y posible abrir una línea de formación específica en sis- temas tanto en lo que se refiere a créditos troncales como, notablemente, a una especialización propia. En lo que concierne a los créditos troncales, es posible y necesario introducir a la enseñanza de la programación en el grado, fundamentalmente orientada a lenguajes de programación usados en Internet y en los sistemas de gestión de bases de datos. También es posible formar en el conocimiento profundo de los estándares informáticos fundamentales para entenderse con los ingenieros informáticos como el Unified Modeling Language (UML) y, claro está, los protocolos y formatos de datos de Internet. $Y$ no hablamos solo de un conocimiento téorico, sino, fundamentalmente, operativo.

\subsection{Resolver el dilema entre formación} generalista y especializada

Articular diversidad y unión podría muy bien ser el reto de nuestros estudios en la próxima década. A diferencia de otras profesiones, las de la información y la documentación conforman una ecología muy compleja; hagamos de esa complejidad una fortaleza en vez de una debilidad.

Para ello, es necesario articular un nuevo modelo educativo con una clara división entre grado y posgrados especializados (García Marco, 2007).

En el grado se debe avanzar en las competencias genéricas (cultura general, idiomas, escribir, presentar, etc.), adquirir competencias específicas instrumentales en el campo del análisis (información textual, visual, estadística, etc.), las tecnologías de la información y comunicación, la programación, el trabajo en equipo y con los clientes y proveedores, la recuperación de información, y una perspectiva completa del mundo de la información actual desde sus aspectos generales hasta los más específicos de los modelos de gestión de la información y la historia de la documentación.

El nivel de posgrado debería preparar para funciones de nivel directivo, para la investigación, la enseñanza universitaria, y, notablemente, para los nichos de empleo que requieren preparación muy específica, difícilmente improvisable: catalogación de documentos, referencia, gestión de registros, documentaciones especializadas (en conjunción con las respectivas especializaciones), administración de sistemas de gestión de contenidos, bibliometría y cienciometría, etc.

Lo que es imposible es hacer bien todo a la vez. Hace falta encarar con una gran dosis de realismo el problema básico: lo que se puede, lo que se quiere y lo que es necesario conseguir; y alcanzar un equilibrio con futuro basado en la división del trabajo. 
Ahora bien, la solución no es llenar de contenidos superficiales los programas. No se trata de intentar enseñar más, sino menos; pero de forma distribuida, usando las posibilidades que ofrece la especialización, especialmente en el nivel de posgrado.

\subsection{Integrar humanidades, tecnología y ciencias sociales de una forma eficaz}

La gestión de servicios es cada vez más un campo en el que se cruzan gestión, tecnología y conocimientos específicos del ciclo de la información. Aspectos importantes como la referencia quedan cada vez más industrializados, de la mano de las grandes bases de datos de referencias y de los grandes buscadores.

Hay que volver a fichar buenos profesores "culturales" para elevar la reflexión y la capacidad de integración de los estudiantes. No tienen por qué ser muchos, pero son absolutamente necesarios.

\subsection{Más teoría, más integración}

Después de muchos años en el que el esfuerzo se ha dedicado a la investigación aplicada, a la investigación docente y a las documentaciones especializadas, es necesario revindicar de nuevo la investigación teórica de carácter omnicomprensivo e integrador. Elaborar un discurso integrado y común a lo largo de las diferentes especialidades y disciplinas es necesario para consolidar una disciplina tan amplia y fraccionada.

\subsection{Necesidad de una discusión basada} en la evidencia

En la línea de los estudios de Delgado, es necesario apoyar la discusión con evidencias y extender la recogida de datos a los diferentes sectores implicados —-profesión, educación, $I+D+I-$ con una perspectiva internacional.

\section{Conclusiones}

En realidad, las últimas tres décadas han sido una época dorada para la profesión, la academia y la enseñanza-aprendizaje en el campo de la información y documentación: los puestos de trabajo se han multiplicado; los profesionales de la información son hoy mucho más valorados y conocidos, como también lo son las unidades de la información; se han multiplicado los puestos de profesor universitario y de ciclos formativos superiores; la formación se ha hecho práctica y se ha tecnologizado... Dentro de este contexto, las personas individuales han podido maximizar sus posibilidades, alejándose incluso de los núcleos centrales e idiosincrásicos de la disciplina, y explorando campos y perspectivas nuevas.

Desde principios de la década, se está produciendo un ajuste de la demanda de estudiantes a la oferta laboral, que, por el momento, sigue creciendo a tasas modestas, entorno al 1,2 \% anual en el sector público. Los nichos clásicos de la disciplina se mantienen firmemente: bibliotecas públicas, bibliotecas universitarias, archivos de la administración... El empleo en la economía privada ha aumentado.

La migración a la infraestructura digital plantea amenazas ciertas, pero por los motivos que he presentado en otro trabajo reciente no va a producir cambios radicales en el empleo en el medio plazo a poco que las unidades de información y documentación se vayan ajustado mínimamente (García Marco, 2008).

Es cierto, sin embargo, que el crecimiento global resulta inferior al que se esperaría de un sector que, en teoría, debería ocupar un lugar fundamental en la sociedad de la información y el conocimiento, lo que debe llevar a una reflexión y un debate serio. No se puede descartar que los procesos de automatización y globalización de la información sean la causa del relativo frenazo que el sector ha experimentado.

Por ello, los datos actuales nos alejan tanto de la depresión como de la euforia. La biblioteconomía y la documentación académicas pueden progresar unidas al desarrollo profesional, pero deben afrontar los cambios y los retos. Para preservar la validez de los programas, hay que continuar los excelentes estudios realizados y es necesario mantener una colaboración estrecha con las asociaciones profesionales y sus líderes, implicándolas profundamente en los procesos de acreditación.

\section{Notas}

(1) La información es también estudiada por otras disciplinas, tanto en sus aspectos teóricos como técnicos. Destacan por la cercanía a la Documentación las ciencias de la comunicación social: periodismo, relaciones públicas, publicidad, etc.

\section{Referencias}

\section{Anuario ThinkEPI (2008) 129-137.}

Åström, F. (2007). Changes in the LIS research front: Timesliced co-citation analyses of LIS journal articles, 19902004. // Journal of the American Society of Information Science \& Technology. 58:7 (2007) 947-957. http://luur. lub.lu. se/luur?func $=$ downloadFile\&fileOld $=576382$

Borrego, Àngel; Comalat, Maite; Estivill Rius, Assumpció (2004). Inserció laboral dels titulats en Biblioteconomia i 
Documentació per la Universitat de Barcelona». BiD: textos universitaris de biblioteconomia i documentación. 12 (juny 2004). http://www2.ub.es/bid/consulta_articulos .php?fichero=12borreg.htm

Cronin, Blaise (2005). An I-dentity crisis? The information schools movement. // International Journal of Information Management. 25 (2005) 363-365.

Delgado López-Cozar, Emilio (2002). La investigación en biblioteconomía y documentación. Gijón: Trea, 2002.

Delgado López-Cozar, Emilio (2003). Las cifras de la documentación en España: 2002. // El profesional de la información. 12:5 (2003) 344-367.

Delgado López-Cozar, Emilio (2007). El ocaso de las enseñanzas universitarias de Documentación en España. // Anuario ThinkEPI (2008) 126-129. Publicado electrónicamente en: IWETEL. ThinkEPI, (16 Oct 2007). http://listserv.rediris.es/cgi-bin/wa?A1=ind0710c\&L=iw etel\&H=1. Extienda la búsqueda a las respuestas buscando "ocaso". Una selección está publicada en el Anuario entre las páginas 129 y 137.

Delgado López-Cozar, Emilio (2007). Las cifras de la infraestructura, profesión y educación documental en España: 2005. // Anuario ThinkEPI. 1 (2007) 207-217.

Delgado-López-Cózar, Emilio; Moneda-Corrochan, Mercedes de la (2008). Las cifras de la enseñanza universitaria en Documentación en España: 2006. //. // El profesional de la información. 17:4 (jul.-ag. 2008) 422-436

Dillon, A. (2007). LIS as a research domain: problems and prospects. I/ Information Research, 12:4. http://InformationR.net/ir/12-4/colis/colis03.html].

Dillon, Andrew and Norris, April (2005). Crying Wolf: An examination and reconsideration of the perception of crisis in LIS. Journal of Education for Library and Information Science 46:4 (2005) 280-298. http://dlist.sir.arizona.edu/1057/01/JELIS.pdf

Eíto Brun, Ricardo (2007). La ingeniería documental: su posible aproximación académica. // El profesional de la información. 16:5 (2007) 490-496.

España. Ministerio de Administraciones Públicas (2008). Gráfico de la evolución del personal al servicio de las administraciones públicas, enero 2008. // Boletín estadístico del personal al servicio de las administraciones públicas: Extracto de la edición impresa de enero 2008. Madrid: Ministerio de Administraciones Públicas, 2008. http://www.map.es/servicios/servicios_on_line/empleo_p ublico/boletin/document_es/Boletin_Estadistico_enero_ 08.pdf

García Marco, F. J. (2007). Perspectivas sobre la enseñanza de posgrado en Ciencias de la Documentación en España. Ibersid, 2007, 291-302.

García Marco, Francisco Javier (2008). El libro electrónico y digital en la ecología informacional. // El profesional de la información. 17:4 (Julio-Agosto 2008) 373-389.

Instituto Nacional de Estadística. http: www.ine.es.

Martínez Méndez, F. J. (1997). La formación en sistemas de información electrónica y nuevos documentos. // Scire, 3(2), 11-30.

Moreiro González, J. A.; Moscoso, P.; Ortiz-Repiso, V. (1995). El mercado de trabajo de los diplomados españoles en biblioteconomía y documentación. // Revista española de documentación científica. 18:4 (1995) 444463.

Moreiro González, José Antonio; et al. (2008). Desarrollo profesional y opinión sobre la formación recibida de los titulados universitarios en información y documentación de las universidades públicas de Madrid (2000-2005). // El Profesional de la Información. 7:3 (May-June 2008) 261-272.
Moscoso, Purificación (2000). Tecnologías y Documentación: veinticinco años de desencuentros en la enseñanza universitaria. // Congreso Universitario de Ciencias de la Documentación. Teoría, historia y metodología de la documentación en España (1975-2000). I. 2000. Madrid: Universidad Complutense, 87-100.

Pérez Agüera, José Ramón (2008). Ingeniería documental frente a artesanía documental: ¿cuál es el modelo a seguir?. // El profesional de la información. 17:3 (2008) 257-260.

Tejada Artigas, C.; Moreiro González, J. A. (2003). Mercado de trabajo en biblioteconomía y documentación: estudios sobre la inserción laboral de los titulados universitarios. // El profesional de la información. 12:1 (enerofebrero, 2003) 4-9. 